\title{
Performance Review System - An ERP Application with Secure Data Storage and Cloud Backup
}

\author{
Aniket Olkar ${ }^{1}$, Nachiket Deo ${ }^{2}$, Vibhav Deo $^{3}$, Prashant Patil ${ }^{4}$ \\ Student, I.T Department, PVPIT, Pune, India ${ }^{1,2,3,4}$
}

\begin{abstract}
Traditionally we have seen that ERP systems are developed using Row-oriented database system. Our project tries to implement column-oriented approach in development of ERP systems. The system which we are trying to develop is Employee Performance Review System. Modern software applications (ERP application in this case) are highly data-centric. As data is highly sensitive security is primary concern, we are trying to increase randomness of the encryption algorithms so as to increase the level of the data security. Loss of important data can affect business process. Hence we provide the data backup using cloud so that in case of application failure the important data needed for business decision making is not lost.
\end{abstract}

Keywords: ERP, Column-Oriented Database, Vertical Partitioning, Symmetric Encryption, Cloud Backup.

\section{INTRODUCTION}

1. ERP: In past two decades the ERP software system have 3.Symmetric Encryption :- Same key is used for gained worldwide acceptance.ERP stands for Enterprise encryption and decryption of data.

Resource Planning. It involves tasks related to various enterprise activities such as production, marketing, finance, sales etc.Various software tools have been developed for assisting the managers, decision makers to help to solve the problems involved in enterprise activities. One of the type of tools is ERP system. The module which under development in this project is Employee Performance Review System. In this module the consultants are rated by four different managers:

1.Incident Manager 2.Change Manager

3.Domain Manager 4.Service Delivery Manager.

The parameters which are used to rate are based on :

1.Productivity and Quality of Delivery.

2.On-Time Delivery. 3.Utilization of Resources.

4.Customer Satisfaction. 5.Team development.

2.Data Security :- Data Security comes into picture while transferring the data over the network. It helps maintain not only the integrity of the data but also confidentiality of the data. In organization, different type of data having various attributes is used to rate an employee. Evaluation of an employee is done based on the input from the respective manager and then the further action of promotion or demotion is decided. Thus, securing the data from an organization is equally important as any other part. It can be done by using various security algorithms.

The model of data security helps in securing the data by encrypting it using an encryption algorithm and is stored into database and then while performing an operation, the required data is extracted, decrypted and then required results can be obtained. The algorithm can be symmetric or asymmetric and depending on the algorithm on which the organization agrees, key is shared which is required for both encryption and decryption.

4.Asymmetric Encryption :- Two different keys are used. One for encryption and other for decryption. Key :- A value used for scrambling the original plaintext and unscrambling the cipher text.

5.Cloud :- Cloud is referred to as, on demand accessing the shared pool of resources over the internet with minimum or no assistance from provider. So basically, you access your data, applications over the internet instead of just using or accessing them locally on your computer's hard drive. Characteristics:

1.On Demand 2.Multitanancy 3.Availability 4.Scalability 5.Security 6.Cost Effective

Role of cloud in this project: Here in this project, cloud is being used as a fail-safe mechanism and as a backup for the data of the application. Data which will be generated by the application will be backed up on the cloud, so in case of an application error or failure of application or possible corruption of data, the work can resume in minimum amount of time and with minimum damage to data.

Cloud Deployment models -

Saas (Software as a service): This is cloud computing model where software is built centrally and made available to client on-demand via a thin client like web browser.

Eg - Mail clients: g-mail, rediffmail

Paas (Platform as a service): Paas is a cloud deployment model where, a development platform is provided as a service to end user. This is mainly for developers. Once you develop you application and deploy it on provider's public cloud, everything from computation to storage of data everything happens at provider end.

Eg - Google app engine, cloud foundry 
Iaas (Infrastructure as a service): In this model, Infrastructure comprising of servers, network, is provided as a service. Instead of buying our own servers and other hardware you can just rent the service from a cloud provider. Eg - Digital Ocean, rack space cloud

6. SSH: Called as Secure Shell, it is used to provide a secure way of communicating between the client and server. SSH is cryptographic protocol which allows clientserver to securely communicate over an untrusted channel. $\mathrm{SSH}$ was designed as a replacement of unsecure shell protocols such as telnet, rsh which send the information such as password in plain text. SSH uses cipher text to send such valuable information as password many application use command line login but remote login can also be used for the same. Two versions of SSH are: 1.SSH-1 2.SSH-2 [8].

\section{LITERATURE REVIEW}

Column-oriented database systems are widely studied in the database community. The main reason is that they perform much more efficiently on analytical workloads than Row-oriented systems. The necessity of modern ERP system is not only to provide reporting but also provide in depth insight of business processes via mathematical analysis of data. So was necessary to evaluate the performance and behaviour of column-oriented system in relation to ERP system [3]. Vertical Partitioned schema has been widely studied. This approach is quite simpler to implement. Inserting data in these schemas is expensive but the main advantage is that the data about a particular entity is available in single table and query has to access only that one table [2]. In order to improve the performance of the joins in column-oriented system it was necessary to study materialization strategies. It involves the time at which the tuples are constructed in a query lifecycle [4]. One such optimized join is the Invisible join which is late-materialized hash join [1]. One of the main area of interest has been data compression. It involves studying various compression techniques like RLE, Bitvector encoding, LZW encoding on a column-oriented system [1].

Cloud computing is vastly beneficial for organizations operation. Cloud's flexibility and low TCO (Total Cost of Ownership) brings tremendous opportunities to enterprise's IT operations. By bringing cloud to ERP domain we are significantly bringing a change in the ERP domain by enhancing the processing capabilities of the organization. Qualitative methods such as systematic literature review and interviews are adopted to execute the research, so data collected through different sources can complement each other [5].

Data Security is a major concern in organization and with increase in attacks on the data, security must be increased. Using the encryption algorithms is not enough but to make it difficult to crack is important. Increasing the randomness in the cipher texts generated for any given data helps in maintaining the data confidentiality. Using an algorithm which randomizes the encryption patterns makes it hard to crack [6].

\section{III.EXISTING SYSTEM}

Existing ERP system follow row-oriented database system. Row-oriented system is highly suitable for update sensitive ERP system. This system is also known as Online Transaction System (OLTP). Whereas the OLAP system which is Online Analytical processing is different from ERP system. As the size of data in ERP system is increasing many firms are trying to outsource to various data warehouse and cloud providers. This increases the TCO (Total cost of Ownership). Also the current ERP system doesn't support various mathematical and analytical processing.

\section{PROPOSED SYSTEM}

The underlying database system which is going to be used is Column-Oriented.

The three Schemas that are widely used are:

1.Vertical Partitioning. 2.Index-only. 3.Materialized view.

Overall structure of the application will be developed using Vertical Partitioning. It involves a foreign key and only one attribute per table. Thus the number of tables will be increased. Materialized View will only be used in cases where the necessary contents of query are known beforehand. Materialized view is used when a particular query is fired many times. Instead of calculating the results each time by accessing the related tables, the results of query are stored in form of a view and when that particular query results are needed the result stored in form of a view is displayed. Thus it prevents recalculation and saves time.

Another thing which we are working on is Data compression. Prior Research work suggests that complex and powerful compression techniques like LZW or Arithmetic Encoding are not useful for database system because database system cannot handle the pressure. So variations of simpler techniques like Bit-vector encoding and Run length encoding will be used and their performance will be tested. The main use of using columnoriented system is for analytical queries. Various types of join like Early materialized, Late materialized and nonMaterialized joins will be used and their performance on column-oriented architecture will be tested.

Late materialization is an approach which does not form tuples until after some part of the query plan has been processed. The early materialization works in exactly opposite way it: it forms tuples as soon as possible. Both Late and Early materialization have pipelined and parallel approach. Our job is to test all these approaches and find out which provides optimal results. One such approach which has been developed in invisible join which works on Star Schema Benchmark (SSBM). Such approach must be followed for vertical partitioned schema [1].

We are not using an actual cloud but we will be simulating one for the purpose of this project we will be using a SSH server-client to communicate with the client application, this server will act as the cloud server which will communicate with the application. 
The encryption algorithm [6] will be used to secure the input data from managers and then the encrypted data will be stored in the database.

\section{SYSTEM WORKING}

Employee performance Review system will have four managers: 1.Incident Manager 2.Change Manager 3.Domain Manager 4.Service Delivery Manager. Each manager will have separate login to Performance Review System. Each manager will fill the data according to respective parameters for each employee. When manager submits the details, the data will be passed to encryption algorithm. Encryption algorithm will encrypt the data and then store it into column-oriented database system. To calculate the final ratings of employee, the data, which is in encrypted format, must be decrypted first and then using appropriate formula the ratings will be calculated. The ratings will be encrypted and stored into ratings table. Once the process is completed the data will be stored on SSH server. Thus the data backup will be created and used in case of application failure.

\section{CONCLUSION}

Use of Column-oriented system with Vertical partitioning schema to develop ERP system will possibly improve the performance .Encryption algorithm used to encrypt sensitive data should improve overall security of the system. Also providing the cloud backup for data will be helpful in case of application failure.

\section{ACKNOWLEDGEMENT}

We would like to thank Prof. S.C.Chaudhari, IT Dept, PVPIT, and express gratitude for supporting us.

\section{REFERENCES}

[1] Daniel J. Abadi, Samuel R. Madden, Nabil Hachem, "ColumnStores vs. RowStores:How Different Are They Really?",db.csail.mit.edu/projects/cstore/abadi-sigmod08.pdf

[2] Daniel J. Abadi, Adam.Marcus, Nabil Hachem, KateHollanbach,"Scalable Semantic Web Data Management Using Vertical Partitioning",dl.acm.org/citation.cfm?id=1325900.

[3] H Plattner,"A Common Database Approach for OLTP and OLAP Using an In-Memory Column Database.", dl.acm.org/citation.cfm?id=1559846, Year 2009.

[4] DJ Abadi ,"Materialization Strategies in a Column-Oriented DBMS", ieeexplore.ieee.org/xpls/abs_all.jsp?arnumber $=4221695$, Year 2007

[5] Shi Jia,"Integrating Conventional ERP System With Cloud Services: From The Perspective Of Cloud Service Type",Royal Institute of Technology, Stockholm University.

[6] Aniket Olkar, "ASCII Based Text Encryption and Decryption With Check For Data Integrity", IJARCCE, Vol. 4, Issue 12, December 2015.

[7] G. Fathima Haseen Raihana, "CLOUD ERP - A SOLUTION MODEL”, IJCSITS, Vol. 2, No. 1, 2012

[8] Wikipedia, Retrieved from https://en.wikipedia.org/wiki/Secure_Shell 